\title{
Rapid inversion of angular deflection data for certain axisymmetric refractive index distributions
}

\author{
R. Rubinstein and P. S. Greenberg
}

\begin{abstract}
Certain functions useful for representing axisymmetric refractive-index distributions are shown to have exact solutions for Abel transformation of the resulting angular deflection data.

Key words: Tomography, Abel inversion.
\end{abstract}

The tomographic reconstruction of axisymmetric refractive-index distributions from angular deflection data (e.g., schlieren, moiré) is a subject of considerable interest, particularly in the areas of combustion science and fluid physics. Although the mathematical solution to this problem was presented by Abel ${ }^{1}$ more than a century ago, the formal solution is seldom used in practice, primarily because of the singular behavior of the integrand at $r=0$ and the requirement for spatially differentiated, often noisy, experimental data. The latter is less of a concern in the present context, since the resulting angular deviations are physically associated with the spatial gradients of the field itself; thus one avoids problems associated with the differentiation of noisy data. A number of schemes exist for the numerical inversion of line-of-sight data, and their properties have been extensively investigated and compared. ${ }^{2}$ We show here that certain classes of function suitable for the representation of axisymmetric refractive-index fields have exact solutions with respect to the Abel inversion of the resulting angular deflection data. These solutions include both Gaussian and Hermite polynomials, the latter being of particular value since Hermite polynomials form an orthonormal basis for radial decomposition. The solutions are physically useful owing to their asymptotic behavior as $r \rightarrow \infty$. Since the solutions are in the form of combinations of known functions and recurrence relations, the inversion reduces to a trivial lookup table operation involv-

R. Rubinstein is with the Lewis Research Center Group, Sverdrup Technology, Inc., Brook Park, Ohio 44142. P. S. Greenberg is with Lewis Research Center, National Aeronautics and Space Administration, Cleveland, Ohio 44135.

Received 8 February 1993; revised manuscript received 7 October 1993 .

0003-6935/94/071141-04\$06.00/0

u 1994 Optical Society of America. ing precomputed coefficients. This process can be implemented extremely rapidly, even when high polynomial orders are retained. In addition the process of basis decomposition with a finite cutoff effectively smooths the data, which diminishes the effects of spatial noise.

When a planar refractive-index distribution $\delta$ is axisymmetric, $\delta=\delta(r)$, and satisfies the conditions

$$
\begin{aligned}
\int_{L}\left|\frac{\mathrm{d} \delta}{\mathrm{d} r}\right| \mathrm{d} s & \ll 1, \\
\delta(r) & \rightarrow 0 \quad \text { for } r \rightarrow \infty,
\end{aligned}
$$

where $L$ denotes any ray path and $\mathrm{d} s$ is the corresponding element of arc length, then the optical path length and the angular deflection along any ray can be evaluated as if the ray did not depart from its initially linear trajectory. Here we use the standard convention $\delta=n-1$, where $n$ is the total value of the refractive index. Condition (1b) means that we denote by $\delta$ the departure of the refractive index from its value in empty space. This approximation leads to the well-known relationships ${ }^{3}$ between angular deflection (e.g., schlieren) data $\epsilon(y)$ and the refractiveindex distribution $\delta(r)$ :

$$
\begin{aligned}
& \epsilon(y)=2 y \int_{y}^{\infty} \frac{\mathrm{d} \delta}{\mathrm{d} r} \frac{\mathrm{d} r}{\left(r^{2}-y^{2}\right)^{1 / 2}}, \\
& \delta(r)=-\frac{1}{\pi} \int_{r}^{\infty} \epsilon(y) \frac{\mathrm{d} y}{\left(y^{2}-r^{2}\right)^{1 / 2}} .
\end{aligned}
$$

Equation (2a) represents the direct problem of evaluating the angular deflection, and Eq. (2b) the inverse problem. Figure 1 illustrates the coordinate system of the initial ray trajectories and their resulting deflections. 


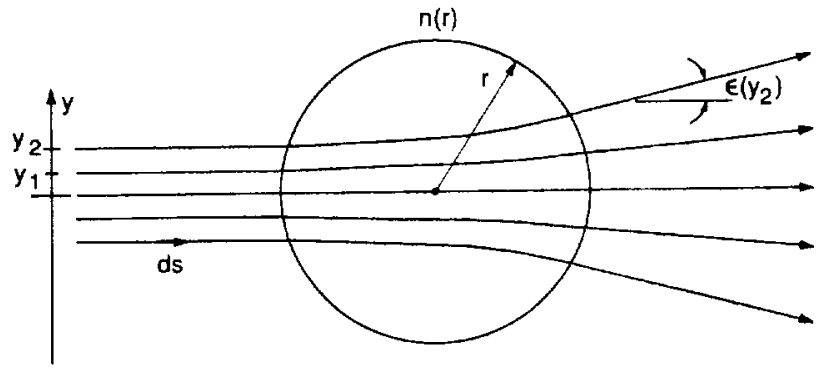

Fig. 1. Geometry of ray deflections in an axisymmetric refractiveindex distribution $n(r): \quad y_{i}$, entrance coordinates of the initial rays; $\mathrm{d} s$, differential length along any given ray; $r$, radial coordinate of the refractive-index distribution; $\epsilon\left(y_{2}\right)$, exit angle of the ray originating at the coordinate $y_{1}=y_{2}$.

A simple pair of functions related by Eqs. (2) is

$$
\int_{x}^{\infty} \nu \exp \left(-v^{2}\right) \frac{d \nu}{\left(v^{2}-x^{2}\right)^{1 / 2}}=\frac{1}{2}(\pi)^{1 / 2} \exp \left(-r^{2}\right)
$$

which follows from elementary changes of the variable in the integral for the gamma function. This equation determines the angular data corresponding to a Gaussian refractive-index distribution,

$$
\delta(r)=A \exp \left(-r^{2}\right)
$$

or the refractive-index distribution when the angular data are of the form

$$
\epsilon(y)=B y \exp \left(-y^{2}\right)
$$

Substitution of either Eq. (4) or (5) into Eq. (2a) or (2b), respectively, reduces at once to Eq. (3).

These results can be generalized by

$$
\begin{aligned}
\int_{x}^{\infty} v^{2 n+1} & \exp \left(-v^{2}\right) \frac{\mathrm{d} v}{\left(v^{2}-x^{2}\right)^{1 / 2}} \\
= & \frac{1}{2}(\pi)^{1 / 2} x^{2 n+1} \exp \left(-x^{2}\right) U\left(\frac{1}{2}, n+\frac{3}{2}, x^{2}\right),
\end{aligned}
$$

where $U$ is a solution of Kummer's equation. ${ }^{4}$ We can evaluate the function $U$ for $n$, an integer, by applying the recurrence relations

$$
\begin{gathered}
\left(c-\frac{3}{2}\right) U\left(\frac{1}{2}, c-1, z\right)+(1-c-z) U\left(\frac{1}{2}, c, z\right) \\
+z U\left(\frac{1}{2}, c+1, z\right)=0 \\
U\left(\frac{1}{2} c+1, z\right)=U\left(\frac{1}{2}, c, z\right)-U^{\prime}\left(\frac{1}{2}, c, z\right),
\end{gathered}
$$

and the initial value

$$
U\left(\frac{1}{2}, \frac{1}{2}, z^{2}\right)=(\pi)^{1 / 2} \exp \left(z^{2}\right) \operatorname{erfc}(z) .
$$

From Eqs. (6) and (7) follow

$$
\begin{aligned}
& U\left(\frac{1}{2}, \frac{3}{2}, z\right)=\frac{1}{2} z^{-1 / 2} \\
& U\left(\frac{1}{2}, \frac{5}{2}, z\right)=\frac{1}{2} z^{-1 / 2}+\frac{1}{4} z^{-3 / 2}
\end{aligned}
$$

These results generalize the stated results above as follows: Define a Gaussian polynomial to be the product of a polynomial and the Gaussian function $\exp \left(-r^{2}\right)$. If the refractive-index distribution is an even Gaussian polynomial,

$$
\delta(r)=\left(a_{0}+a_{2} r^{2}+\cdots\right) \exp \left(-r^{2}\right),
$$

its derivative is an odd Gaussian polynomial, and the corresponding angular data are the odd Gaussian polynomial found by substitution of the results of Eqs. (6)-(9) into Eq. (2a). Conversely, if the angular data are a given odd Gaussian polynomial, the corresponding even Gaussian polynomial refractive-index distribution follows by substitution of the results of Eqs. (6)-(9) in Eq. (2b).

These exact results imply a simple approximation scheme when the angular data can be accurately represented by an odd Gaussian polynomial; because of the asymptotic return of the refractive index to its reference value in many combustion science and fluid physics applications, this approximation is reasonable. It is necessary first to approximate the angular data by an odd Gaussian polynomial. The orthogonality of Hermite polynomials makes this process straightforward, namely,

$$
\epsilon(y) \sim \sum_{i \text { odd }} a_{i} H_{i}(y) \exp \left(-y^{2}\right)
$$

with

$$
a_{i}=\int_{-\infty}^{\infty} \frac{\epsilon(y) H_{i}(y)}{(\pi)^{1 / 2} i ! 2^{i}} .
$$

Inspection of Eqs. (2) demonstrates that the refractiveindex distribution is given by an even Gaussian polynomial.

The recurrence relation [Eqs. (7)] can be applied to expedite the calculation. Since the right-hand side of Eq. (6) is a Gaussian polynomial, we define

$$
x^{2 n+1} U\left(\frac{1}{2}, n+\frac{3}{2}, x^{2}\right)=V\left(n, x^{2}\right) .
$$

Then Eqs. (7) provide the recurrence

$$
\begin{aligned}
V\left(n, x^{2}\right)= & \left(x^{2}+n-\frac{1}{2}\right) V\left(n-1, x^{2}\right) \\
& +x^{2}(1-n) V\left(n-2, x^{2}\right) .
\end{aligned}
$$

We define $H_{n, m} b y$

$$
H_{n}(x)=\sum_{m \geq 0} H_{n, m} x^{m} .
$$


Explicitly

$$
\begin{aligned}
& H_{0,0}=1, H_{0, m}=0 \quad \text { for } m \geq 1, \\
& H_{1,0}=0, H_{1,1}=2, H_{1, m}=0 \quad \text { for } m \geq 2, \\
& H_{n, 0}=-2(n-1) H_{n-2,0} \quad \text { for } n \geq 2, \\
& H_{n, m}=-2(n-1) H_{n-2, m}+2 H_{n-1, m-1} \\
& \quad \text { for } n \geq 2, m \geq 1 .
\end{aligned}
$$

Then, writing

$$
\begin{aligned}
\epsilon(y)=\sum_{n \text { odd }} a_{n} H_{n}(y) & \exp \left(-y^{2}\right) \\
& =\sum_{n \text { odd }, p \geq 0} a_{n} H_{n, 2 p+1} y^{2 p+1} \exp \left(-y^{2}\right),
\end{aligned}
$$

we give the corresponding refractive index as

$$
\begin{aligned}
\delta(r)= & -\frac{1}{\pi} \int_{r}^{\infty} \frac{\mathrm{d} y}{\left(y^{2}-r^{2}\right)} \sum_{n \text { odd, } p \geq 0} a_{n} H_{n, 2 p+1} y^{2 p+1} \exp \left(-y^{2}\right) \\
= & -\frac{1}{2(\pi)^{1 / 2}} \sum_{n \text { odd }, p \geq 0} v^{2 p+1} \\
& \times \exp \left(-v^{2}\right) U\left(\frac{1}{2} p+\frac{3}{2}, v^{2}\right) H_{n, 2 p+1} \\
= & -\frac{(\pi)^{1 / 2}}{2} \sum_{n \text { odd }, p \geq 0} a_{n} H_{n, 2 p+1} \exp \left(-v^{2}\right) V\left(p, v^{2}\right),
\end{aligned}
$$

which we can rapidly and accurately evaluate to any order by using Eqs. (12) and (13).

These results are stated here in terms of dimensionless variables. In inverting experimental data the variables should be scaled so that the decay of the Hermite polynomials appropriately matches the decay of the data.

As a representative application we reconstructed the refractive-index distribution

$$
\delta(r)=\exp \left[-(r-1)^{2}\right]+\exp \left[-(r+1)^{2}\right]
$$

using seven-term Hermite interpolation of corresponding numerically evaluated angular data. The results in Fig. 2 show that the reconstruction is very accurate. One can reduce the error in the reconstruction further simply by taking higher-order Hermite approximations of the data. Because the approximation requires functions that are evaluated by simple recurrence relations and coefficients that are evaluated by simple numerical quadratures, the computer resources required by the inversion are negligible.

An advantage of this procedure over direct numerical Abel inversion is that least-squares curve fitting is a smoothing process that reduces the noise sensitivity of the computation. ${ }^{5}$ To bound the effect of noise on this inversion, we establish the inequality

$$
\int_{0}^{\infty}\left|V\left(n, x^{2}\right) \exp \left(-x^{2}\right)\right| \mathrm{d} x \leq C \int_{0}^{\infty} v^{2 n+1} \exp \left(-\nu^{2}\right) \mathrm{d} \nu
$$

$$
n(r)=(1 / 2)\left\{e^{-(r-1) * * 2}-e^{-(r+1) * 2}\right\}
$$

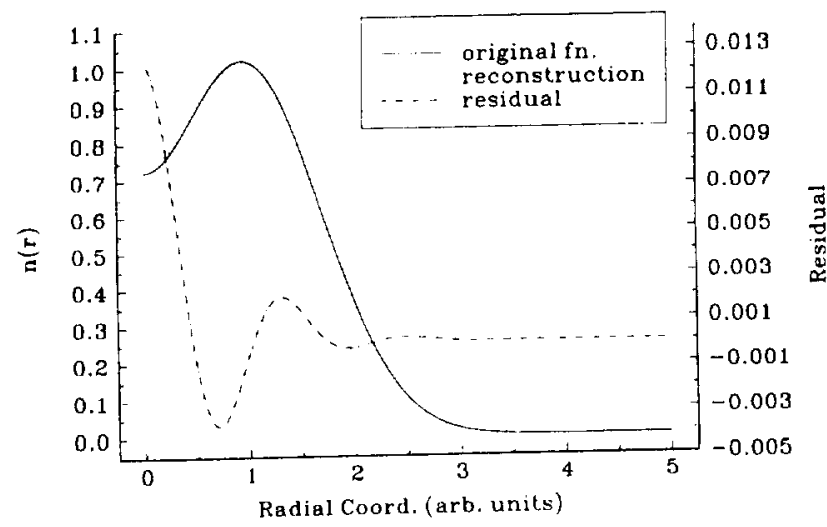

Fig. 2. Error in seven-term reconstruction of a model refractiveindex field.

with $C$ independent of $n$. Therefore noise in the inversion is then bounded by noise in the data; specifically the magnitude of the noise does not grow if the number of terms used in the inversion is increased.

Note the equality

$$
\int_{0}^{\infty} v^{2 n+1} \exp \left(-v^{2}\right) \mathrm{d} v=\frac{1}{2} n !
$$

and the estimate

$$
\int_{0}^{\infty} \nu^{2 n} \exp \left(-v^{2}\right) \mathrm{d} v \sim n !
$$

Let

$$
V\left(n, x^{2}\right)=a_{0} x^{2 n}+a_{1} x^{2 n-2}+\cdots+a_{2 n} .
$$

Then the recurrence formula given by Eq. (12) provides the bound

$$
\left|a_{p}\right| \leq 3(2 p-1) ! ! / 2^{p+1} \sim 3 p ! \quad \text { for } p \geq 1,
$$

where as usual $(2 p-1) ! !=(2 p-1)(2 p-3), \ldots, 1$.

Therefore

$$
\begin{aligned}
& \int_{0}^{\infty}\left|V\left(n, x^{2}\right) \exp \left(-x^{2}\right) \mathrm{d} x\right| \int_{0}^{\infty} v^{2 n+1} \exp \left(-v^{2}\right) \mathrm{d} v \\
& \leq 6 \pi^{1 / 2}\left[\sum_{p \geq 1} \frac{(2 p-1) ! !}{2^{p+1}} \frac{(2 n-2 p-1) ! !}{n ! 2^{2 n-2 p}}+1\right] \\
& \sim 6 \pi^{1 / 2}\left[\sum_{p \geq 1} p !(n-p) ! / n !+1\right] .
\end{aligned}
$$

The inequality

$$
\sum_{0 \leq p \leq n} p !(n-p) ! / n ! \leq \frac{8}{3}
$$

then establishes inequality (14). Inequality (15) has been evaluated numerically and is shown to peak at 
its maximum value for $n=3$ and 4 , from which point it decreases monotonically with increasing $n$.

\section{References}

1. N. H. Abel, "Auflosung einer mechanischen Aufgabe," J. Reine Angew. Math. 1, 153-157 (1826).

2. C. J. Dasch, "One-dimensional tomography: a comparison of Abel, onion-peeling, and filtered backprojection methods, "Appl. Opt. 31, 1146-1152 (1992).
3. C. M. Vest, "Interferometry of strongly refracting axisymmetric phase objects," Appl. Opt. 14, 1601-1606 (1975).

4. A. Erdelyi, W Magnus, F. Oberhettinger, and F. Tricomi, Higher Transcendental Functions (McGraw-Hill, New York, 1953), Vol. 1.

5. B. J. Hughey and D. A. Santavicca, "A comparison of techniques for reconstructing axisymmetric reacting flow fields from absorption measurements," Combust. Sci. Technol. 29, 167-190 (1982). 